\title{
Experimental Periodontitis Results in Prediabetes and Metabolic Alterations in Brain, Liver and Heart: Global Untargeted Metabolomic Analyses
}

Keywords: Prediabetes; Global metabolomic analysis; Experimental periodontitis

\begin{abstract}
Results from epidemiological studies suggest that there is an association between periodontitis and prediabetes, however causality is not known. The results from our previous studies suggest that induction of periodontitis leads to hyperinsulinemia glucose intolerance and insulin resistance, all hallmarks of prediabetes. However, global effects of periodontitis on critical organs in terms of metabolic alterations are unknown.

We determined the metabolic effects of periodontitis on brain, liver heart and plasma resulting from Porphyromonas gingivalis induced periodontitis in mice. Periodontitis was induced by oral application of the periodontal pathogen, Porphyromonas gingivalis for 22 weeks. Global untargeted biochemical profiles in samples from these organs/ plasma were determined by liquid and gas chromatography/mass spectrometry and compared between controls and animals with periodontitis.
\end{abstract}

Oral application of Porphyromonas gingivalis induced chronic periodontitis and hallmarks of prediabetes. The results of sample analyses indicated a number of changes in metabolic readouts, including changes in metabolites related to glucose and arginine metabolism, inflammation and redox homeostasis. Changes in biochemicals suggested subtle systemic effects related to periodontal disease, with increases in markers of inflammation and oxidative stress most prominent in the liver. Signs of changes in redox homeostasis were also seen in the brain and heart. Elevated bile acids in liver were suggestive of increased biosynthesis, which may reflect changes in liver function. Interestingly, signs of decreasing glucose availability were seen in the brain. In all three organs and plasma, there was a significant increase in the microbiome-derived bioactive metabolite 4-ethylphenylsulfate sulfate in animals with periodontitis.

The results of metabolic profiling suggest that periodontitis/ bacterial products alter metabolomic signatures of brain, heart, liver, and plasma in the prediabetic state. These data provide scientific community valuable metabolic signatures that become the basis for understanding the impact of periodontitis on a systemic disease and potentially targets for therapeutic intervention.

\footnotetext{
Abbreviations

Pg: Porphyromonas gingivalis; HI: hyperinsulinemia; GI: glucose intolerance; IR: insulin resistance; T2DM: Type 2 Diabetes Mellitus; CEJ: cemento-enamel junction; HOMA: Homeostatic model assessment; ipGTT: intraperitoneal glucose tolerance test; 4-EPS 4-ethylphenylsulfate
}

\section{Journal of Oral Biology}

\author{
Vladimir Ilievski' ${ }^{1}$, Jason M Kinchen ${ }^{2}$, Ramya \\ Prabhu $^{3}$, Fadi Rim ${ }^{3}$, Lara Leoni ${ }^{4}$, Terry G. \\ Unterman $^{5}$ and Keiko Watanabe ${ }^{1^{*}}$
}

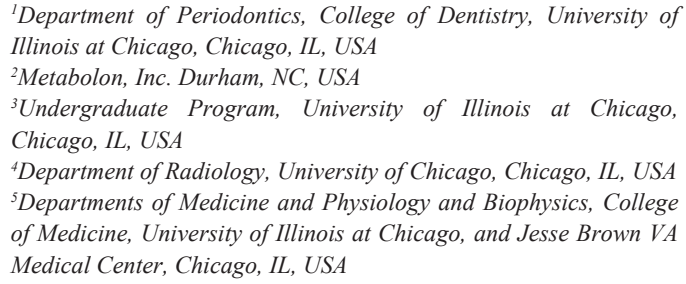

\section{*Address for Correspondence}

Keiko Watanabe, Department of Periodontics, College of Dentistry, University of Illinois at Chicago, Chicago, IL, USA, Tel: 312-996-0636; Fax: 312-996-0943; E-mail: keiko@uic.edu

\section{Submission: 22 March, 2015}

Accepted: 18 April, 2016

Published: 23 April, 2016

Copyright: ( 2016 llievski V, et al. This is an open access article distributed under the Creative Commons Attribution License, which permits unrestricted use, distribution, and reproduction in any medium, provided the original work is properly cited.

\section{Introduction}

Epidemiological and clinical studies have identified a clinically important association between periodontitis (gum disease with associated loss of tooth supporting bone) and Type 2 Diabetes Mellitus (T2DM) [1,2]. Results from epidemiological studies also suggest a close association between periodontitis and both prediabetes and metabolic syndrome [3]. However, a causal relationship between periodontitis and prediabetes has not been established.

A major limitation in determining causality is that experimental induction of destructive periodontal disease (periodontitis) is clearly not acceptable in humans. In previous studies, we have established animal models of induced periodontitis and studied the causality linking periodontitis to the development of glucose intolerance (GI), hyperinsulinemia (HI), and insulin resistance (IR), all hallmarks of prediabetes. Results from our previous studies demonstrate that animals with LPS/bacterial product-induced periodontitis develop prediabetes $[4,5]$ and that bacterial products/LPS are an important initiator of HI, GI, and IR [5]. However, the global impact of periodontitis on metabolism in organs distant from the oral cavity has not been investigated.

Metabolomics is the systematic study of the unique biochemical fingerprint (metabolic signature) that results from specific cellular processes [6] and is one of the core disciplines of systems biology [7]. The metabolome is the collective signature of all metabolites in a biological cell, tissue, organ or organism, which are the intermediate and end products of cellular processes [8]. Thus, sample specific metabolomes from saliva, plasma, heart, liver etc. can be analyzed to determine disease effects locally and systemically [9-11]. Metabolomics is a powerful tool to identify alterations in specific metabolic pathways that result from disease with the potential to map early biochemical changes in pathophysiology, identify predictive 
Citation: Ilievski V, Kinchen JM, Prabhu R, Rim F, Leoni L, et al. Experimental Periodontitis Results in Prediabetes and Metabolic Alterations in Brain, Liver and Heart: Global Untargeted Metabolomic Analyses. J Oral Bio. 2016; 3(1): 12.

ISSN: 2377-987X

biomarkers as well as identify targets that can be exploited for early prevention and/or treatment of the disease [12].

Only a limited number of studies have focused on identifying differences in metabolic profiles between periodontitis and control subjects. These studies focused on salivary biochemical $[9,13,14]$ and reported various differences in metabolites in these fluids. These differences included dipeptide, amino acid, carbohydrate, lipid, and nucleotide metabolites consistent with the degradation of macromolecules including proteins, triacylglycerol, glycerolphospholipids, polysaccharides, and polynucleotides, which may reflect the outcome of host-bacterial interactions [13] The differences in the biochemical profiles between healthy and periodontitis saliva samples in non-diabetic subjects also included increased levels of markers of cellular metabolic stress and increased purine catabolism and glutathione metabolism [9]. However, how periodontitis/periodontal pathogen impacts metabolism in organs distant from the oral cavity has not been investigated. In this study we determined the effects of oral application of Porphyromonas gingivalis $(\mathrm{Pg})$ on metabolism in critical organs (brain, liver, heart), and plasma, using untargeted global metabolomic profiling of control animals and animals with oral Pg application. These data provide the first systematic comprehensive overview of metabolic changes that occur as a result of oral application of a periodontal pathogen and provide a valuable resource of candidate metabolites and metabolic pathways to the scientific community to undertake further studies. Future studies will lead to the potential for development of strategies to prevent periodontal pathogen induced metabolic disturbances such as development of prediabetes, dementia, atherosclerosis, and non-alcoholic fatty liver disease with which periodontitis has been associated.

\section{Materials and Methods}

\section{Animals}

This study was carried out in strict accordance with the recommendations outlined in the Guide for the Care and Use of Laboratory Animals of the National Institutes of Health. The protocol was approved by the Institutional Animal Care and Use Committee at the University of Illinois at Chicago (Protocol approval \#12-152).

Twenty 6-week old male C57BL/6 mice were purchased from Jackson Laboratories (Bar Harbor, ME). Following one-week acclimatization, mice were maintained on regular chow (7912 Teklad LM-485) (Envigo RMS, Indianapolis, IN) and water ad libitum at a constant temperature $\left(22^{\circ} \mathrm{C}\right)$ with humidity of $45 \%$ to $55 \%$ in a 12 hour light/dark cycle.

\section{Bacterial culture and preparation}

Porphyromonas gingivalis (strain W83) was grown anaerobically $\left(85 \% \mathrm{~N}_{2}, 10 \% \mathrm{H}_{2}\right.$ and $\left.5 \% \mathrm{CO}_{2}\right)$ in GasPak anaerobic containers (Becton Dickinson (BD), Franklin Lakes, NJ) using GasPak EZ pouches (BD) at $37^{\circ} \mathrm{C}$ in sterile $3 \%$ Bacto Todd Hewitt Broth (BD) (pH 7.4) supplemented with $0.001 \%$ hemin and $0.0001 \%$ vitamin K1 (both from Sigma, St. Louis, MO). Bacteria were grown overnight and approximate cell density was determined by spectrophotometry at an optical density of $550 \mathrm{~nm}$, based on a standard curve established by colony formation on bacterial plates. The density of the culture was adjusted to an O.D. of $\sim 1.5\left(2 \mathrm{X} 10^{9}\right.$ cells/ ml). Appropriate amounts of culture containing $1 \times 10^{9}$ cells were transferred to 10 microfuge tubes, vortexed briefly and centrifuged at 10,000 $\mathrm{g}$ for 2 minutes at $4{ }^{\circ} \mathrm{C}$. The supernatants were discarded and the pellets were re-suspended in $4{ }^{\circ} \mathrm{C}$ PBS and repelleted by centrifugation. The supernatants were removed and bacteria re-suspended in $100 \mu \mathrm{l}$ of $2 \%$ carboxymethyl cellulose in PBS and immediately placed on ice until used for oral application in mice.

\section{Study design}

Animals were divided into 2 groups ( $\mathrm{n}=10$ per group): $100 \mathrm{ul}$ of Pg in carboxymethylcellulose was applied (2 applications of $50 \mathrm{ul}$ ) in the oral cavity every other day (Monday, Wednesday, and Friday) for 22 weeks (experimental group) and carboxymethylcellulose vehicle alone was applied in the oral cavity (control group). Mice that received oral applications of Pg were kept in a separate room (Biohazard Room) from control mice to avoid cross contamination. All animals were weighed once a week for the duration of the experiment. Mice were sacrificed during the $23^{\text {rd }}$ week. A portion of liver, brain, and the entire heart and a sample of plasma from each animal were snap frozen in liquid nitrogen and shipped to Metabolon, Inc. (Durham, NC) for analyses of metabolic products. Maxillae were used to assess bone loss by microtomography as described below. Individuals performing examination of bone loss and metabolic analyses were blinded to the group to which individual animals were assigned.

\section{Measurement of alveolar bone loss by microtomography}

To assess alveolar bone loss, image acquisition was performed at the Integrated Small Animal Imaging Research Resource (iSAIRR) at the University of Chicago, Chicago, IL. Maxillae were scanned on a Trifoil GMI Triumph (Northridge, CA) with the following parameters: voltage: $60 \mathrm{k}$; V current: $140 \mathrm{uA}$; field of view: 28.16 $\mathrm{mm}$; projections: 1024; pixel size: $54 \mathrm{~mm}$. AMIRA software (FEI, Hillsboro, OR) was used for image analysis. Maxillae were rendered in $3 \mathrm{D}$ and cross sections cut through the distal root of $1^{\text {st }}$ molars were visualized and linear measurements were taken in millimeters from the cemento-enamel junction (CEJ) to the alveolar bone crest to assess bone loss.

\section{Intraperitoneal glucose tolerance test (ipGTT)}

ipGTTs were performed at baseline and at 22 weeks post-initiation of Pg application. Briefly, following a $14 \mathrm{hr}$ fast and collection of blood samples to determine glucose and insulin levels, $50 \%$ dextrose ( $2 \mathrm{~g} / \mathrm{kg}$ body weight) was administered intraperitoneally and glucose levels in tail blood were determined after 15, 30, 60, 90 and $120 \mathrm{~min}$ using a glucometer.

\section{Metabolomic profiling}

Metabolomic and statistical analyses were conducted at Metabolon, Inc. (Durham, NC) as described previously [15]. Briefly, tissue samples from control and periodontitis groups ( $\mathrm{n}=9$ animals per group) were homogenized, subjected to methanol extraction and then split into aliquots for analyses by ultrahigh performance liquid chromatography/mass spectrometry (UHPLC/MS) in the positive (two methods, one optimized for hydrophilic, the other hydrophobic compounds), negative or polar ion mode. Metabolites were identified 


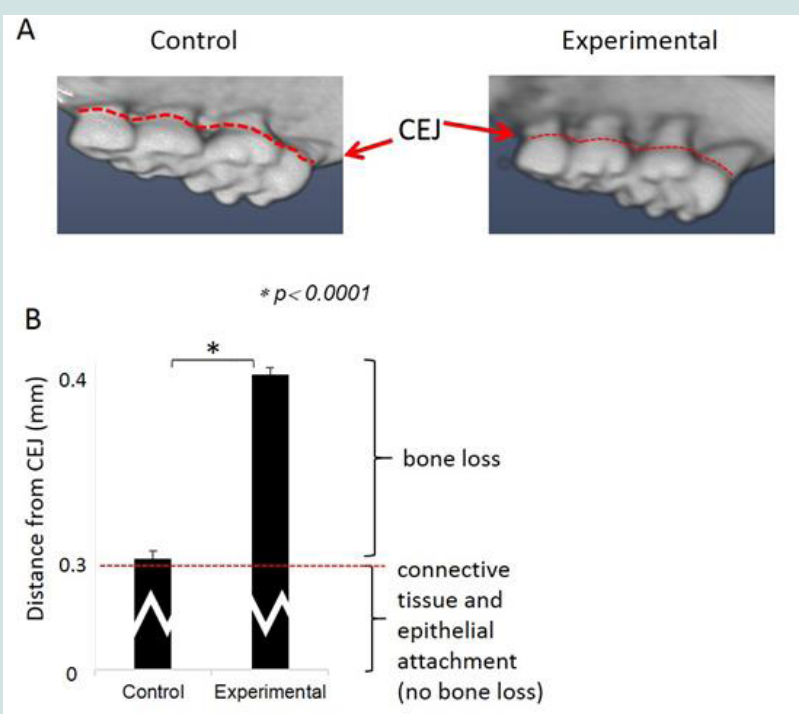

Figure 1: Oral application of Pg induces alveolar bone loss.

A) Image from micro-tomography. B) Alveolar bone loss. The bracket on the right bottom indicates the mean distance where epithelia and connective tissue attach thus not considered as bone loss. The bracket on the top indicates the mean bone loss. (mean+SD). Periodontitis: oral application of Pg, Control: vehicle alone.

by automated comparison of ion features to a reference library of chemical standards followed by visual inspection for quality control. For quality assurance/control, a pooled matrix (or for plasma, an internal matrix) as well as several internal standards were assessed to determine instrument variability, with relative standard deviation; at $4 \%$ (plasma) or $2 \%$ (brain, liver, and heart).

\section{Statistical analysis}

For statistical analyses and data display, any missing values were assumed to be below the limits of detection; these values were imputed with the compound minimum (minimum value imputation). Welch's two-sample $t$-test was used to identify biochemical that differed significantly between groups. Mann-Whitney test was used to assess differences in ipGTT, glucose, insulin and HOMA-IR for control and periodontitis groups. $\mathrm{P}<0.05$ was considered as a significant difference between periodontitis and control samples with a trend value of $0.05<\mathrm{p}<0.1$ as approaching significance.

\section{Results}

Oral application of $\mathrm{P}$. gingivalis induced periodontitis, glucose intolerance, hyperinsulinemia and insulin resistance

Repeated oral application of Pg induced significant alveolar bone loss compared to control animals (Figure 1). The results from ipGTT at week 22 revealed impaired glucose tolerance in Pgtreated mice, with hyperglycemia persisting for up to 120 min postglucose load in mice with periodontitis as evidenced by $\mathrm{p}$ values at each time point (Table 1). Hyperinsulinemia and insulin resistance were also observed, however, fasting glucose levels were within the normal range (Table 2). Thus, oral application of Pg resulted in the development of prediabetes.

Global metabolomic analyses identified a number of biochemicals which were significantly altered by oral application of Pg

Following data acquisition and curation, a total of 552 biochemicals were identified in plasma, 442 in brain, 576 in liver, and 580 in heart. Following log transformation and imputation of missing values, if any, with the minimum observed value for each compound, Welch's two-sample $t$-test was used to identify biochemicals that differed significantly between groups. In plasma, the levels of 24 biochemicals were significantly different between groups $(\mathrm{p}<0.05)$; 4 metabolites were increased and 20 decreased. In brain, there were 29 biochemicals with significant alterations between groups; 6 were increased and 23 were decreased. Liver had 43 biochemicals with significant alterations: 40 were increased and 3 were decreased. In heart, 23 biochemicals were significant altered between groups of which 20 were increased and 3 were decreased (Table 3 ).

In addition, there were a similar number of biochemicals which achieved a trend value $(0.05<\mathrm{p}<0.10)$ toward significance in these analyses (Table 3).

\section{Oral application of $\mathrm{Pg}$ induced changes in glucose metabolism in the brain.}

Glucose is utilized to support a variety of physiological processes, including energy generation, fatty acid synthesis, protein glycosylation, and nucleotide biogenesis. Glucose is the obligatory

Table 1: Glucose tolerance test results at week 22 in control and periodontitis mice ( $n=10$ animals per group). Data presented are mean+S.D. Mann-Whitney test.

\begin{tabular}{|c|c|c|c|}
\hline Time (min.) & Control (mg/dL) & $\begin{array}{c}\text { Experimental } \\
(\mathbf{m g} / \mathbf{d L})\end{array}$ & $p$ value \\
\hline 0 & $171.4 \pm 34.6$ & $142.5 \pm 27.8$ & 0.049 \\
\hline 15 & $408.7 \pm 73.1$ & $499.7 \pm 80.6$ & 0.023 \\
\hline 30 & $436.6 \pm 92.84$ & $529.3 \pm 67.9$ & 0.037 \\
\hline 60 & $360.5 \pm 105.6$ & $510.1 \pm 109.4$ & 0.019 \\
\hline 90 & $269.4 \pm 83.3$ & $524.7 \pm 88.2$ & 0.0002 \\
\hline 120 & $174 \pm 24.4$ & $441.3 \pm 103.1$ & $3.41 \mathrm{E}-05$ \\
\hline
\end{tabular}


Citation: Ilievski V, Kinchen JM, Prabhu R, Rim F, Leoni L, et al. Experimental Periodontitis Results in Prediabetes and Metabolic Alterations in Brain, Liver and Heart: Global Untargeted Metabolomic Analyses. J Oral Bio. 2016; 3(1): 12.

Table 2: Fasting glucose, insulin levels and HOMA-IR at week $22(n=10$ animals per group). Data presented are mean+S.D. Mann-Whitney test.

\begin{tabular}{|c|c|c|c|}
\hline & Control & Experimental & $\boldsymbol{p}$ value \\
\hline $\begin{array}{c}\text { Fasting glucose } \\
\text { (mg/dL) }\end{array}$ & $182 \pm 39.5$ & $150.2 \pm 24.9$ & 0.08 \\
\hline Insulin $(\boldsymbol{\mu g} / \mathbf{L})$ & $0.4 \pm 0.2$ & $0.8 \pm 0.3$ & 0.03 \\
\hline HOMA-IR & $3.9 \pm 2.5$ & $8.1 \pm 1.7$ & 0.03 \\
\hline
\end{tabular}

Table 3: Summary of biochemical alterations induced by Pg application (experimental group) from a total of 552 named biochemicals identified in plasma, 442 in brain, 576 in liver and 580 in heart. Red arrow indicates the number of biochemicals increased. Green arrow indicates the number of biochemicals decreased. Experimental: oral application of $\mathrm{Pg}$, Control: vehicle alone.

\begin{tabular}{|c|c|c|c|c|}
\hline \multirow{4}{*}{ Biochemical Alterations } \\
\hline Welch's Two-Sample $t$-Test & \multicolumn{4}{|c|}{ Experimental } \\
\cline { 2 - 5 } & Plasma & Brain & Liver & Heart \\
\hline Total biochemicalsp $\leq 0.05$ & 24 & 29 & 43 & 23 \\
\hline $\begin{array}{c}\text { Biochemicals } \\
(\uparrow \downarrow)\end{array}$ & $4 / 20$ & $6 / 23$ & $40 / 3$ & $20 / 3$ \\
\hline $\begin{array}{c}\text { Total biochemicals } \\
\text { 0.05<p<0.10 }\end{array}$ & 25 & 24 & 42 & 24 \\
\hline $\begin{array}{c}\text { Biochemicals } \\
(\uparrow \downarrow)\end{array}$ & $8 / 17$ & $10 / 14$ & $38 / 4$ & $19 / 5$ \\
\hline
\end{tabular}

energy substrate of the adult brain. In the brain, decreases in glucose $(\mathrm{p}=0.093)$, glucose 6-phosphate $(\mathrm{p}=0.0299)$, and fructose6-phosphate ( $\mathrm{p}=0.0338)$ suggest changes in glucose metabolism or glucose availability in animals with periodontitis. Pentose phosphate metabolites (6-phosphogluconate and sedoheptulose-7-phosphate) ( $\mathrm{p}=0.0159$ and $\mathrm{p}=0.0611$ respectively), the hexosamineglucosamine6-phosphate $(\mathrm{p}=0.0152)$, and the glycolytic end-product pyruvate
( $\mathrm{p}=0.0802$ ), were also decreased, which would be consistent with decreasing glucose availability in animals with periodontitis. Collectively, these data suggest that glucose utilization is relatively reduced in the brain of animals with periodontitis compared to controls (Figure 2 and Table 4).

\section{Oral application of Pg induced changes in other biochemicals in the brain.}

Between group analyses also revealed changes in redox homeostasis in brain: for example, decrease in anserine (a dipeptide metabolite of histidine with anti-oxidant function) $(\mathrm{p}=0.0649)$ (Table $4)$.

The benzoate metabolite 4-ethylphenylsulfate (4-EPS), which is derived from liver modifications of a microbiome-derived tyrosine metabolite 4-ethylphenol, was marginally increased in the brains of animals with Pg application $(\mathrm{p}=0.0574)$ compared to control animals.

Analysis also revealed a decrease in several arginine-derived biochemicals such as citrulline in the brain $(p=0.0264)$ in animals with periodontitis compared to control animals. In addition, ornithine $(\mathrm{p}=0.078)$ and acetylated derivatives of arginine and ornithine, $\mathrm{N}$-acetylarginine and $\mathrm{N}$-delta-acetylornithine $(\mathrm{p}=0.00118$, $\mathrm{p}=0.075$ respectively), creatinine $(\mathrm{p}=0.004)$, and the polyamines, spermine $(\mathrm{p}=0.032)$ and spermidine $(\mathrm{p}=0.0059)$, were also decreased in animals with periodontitis compared to control animals (Table 4). While arginine was not decreased in the dataset, decreases in related metabolites could suggest limiting availability. We also observed elevated levels of 1,5-anhydroglucitol (1,5-AG) ( $\mathrm{p}=0.0093)$, a naturally occurring monosaccharide, in the brains of animals with periodontitis compared with controls.

Finally, lysolipid is significantly increased in the brain of animals with Pg application $(\mathrm{p}=0.0315)$.

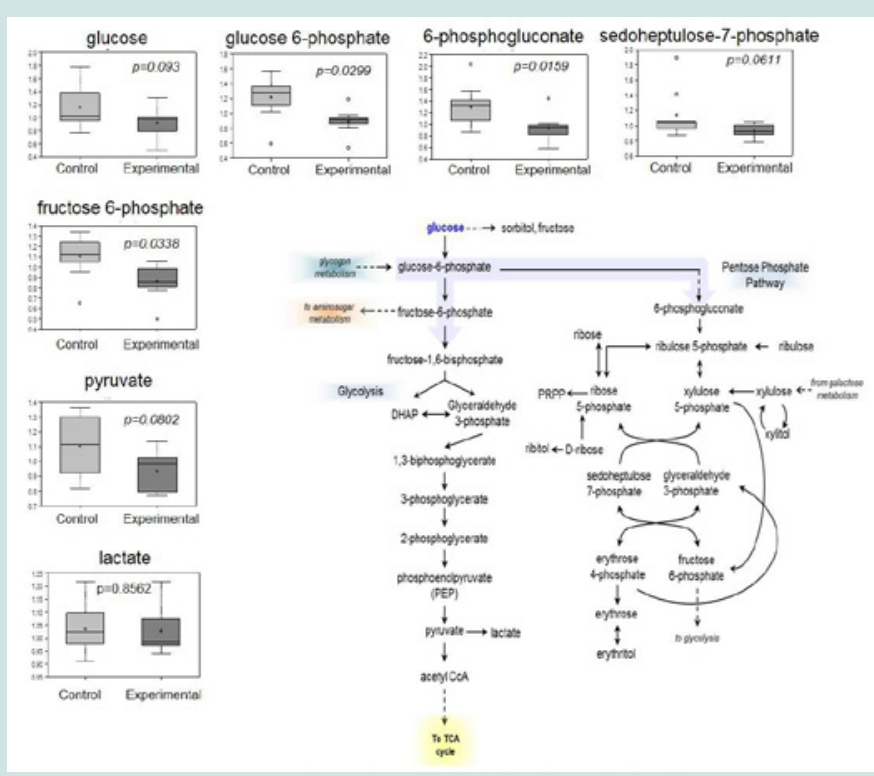

Figure 2: Glucose metabolism in brain of control and experimental animals.

Glucose levels tend to be low in the brain of mice with periodontitis. Glucose utilization is significantly lower in these animals compared to controls. Data presented are mean+S.D. Welch's two-sample t-test. $\mathrm{N}=9$ animals per group. Experimental: oral application of $\mathrm{Pg}$, Control: vehicle alone. 
Citation: Ilievski V, Kinchen JM, Prabhu R, Rim F, Leoni L, et al. Experimental Periodontitis Results in Prediabetes and Metabolic Alterations in Brain, Liver and Heart: Global Untargeted Metabolomic Analyses. J Oral Bio. 2016; 3(1): 12.

ISSN: $2377-987 X$

Table 4: Metabolic differences in the brain of experimental vs control animals. Fold change is expressed as Experimental/Control. $\mathrm{N}=9$ animals per group.

\begin{tabular}{|c|c|c|c|c|}
\hline Super Pathway & Sub Pathway & Biochemical Name & $\begin{array}{l}\text { Fold } \\
\text { changes }\end{array}$ & p-value \\
\hline \multirow{10}{*}{ Amino Acid } & Creatine Metabolism & creatinine & 0.91 & 0.0004 \\
\hline & Urea cycle; Arginine and Proline Metabolism & $\mathrm{N}$-acetylarginine & 0.87 & 0.0018 \\
\hline & \multirow{2}{*}{ Polyamine Metabolism } & spermine & 0.17 & 0.0032 \\
\hline & & spermidine & 0.34 & 0.0059 \\
\hline & Lysine Metabolism & N2-acetyllysine/N6-acetyllysine & 0.89 & 0.009 \\
\hline & Phenylalanine and Tyrosine Metabolism & O-methyltyrosine & 0.82 & 0.0152 \\
\hline & \multirow{2}{*}{ Urea cycle; Arginine and Proline Metabolism } & citrulline & 0.84 & 0.0264 \\
\hline & & urea & 0.8 & 0.0275 \\
\hline & \multirow{2}{*}{ Glutamate Metabolism } & S-1-pyrroline-5-carboxylate & 0.77 & 0.0399 \\
\hline & & glutamate, gamma-methyl ester & 0.88 & 0.0498 \\
\hline \multirow{4}{*}{ Carbohydrate } & Aminosugar Metabolism & glucosamine-6-phosphate & 0.84 & 0.0152 \\
\hline & \multirow{3}{*}{$\begin{array}{c}\text { Glycolysis, Gluconeogenesis, and Pyruvate } \\
\text { Metabolism }\end{array}$} & 1,5-anhydroglucitol (1,5-AG) & 1.18 & 0.0173 \\
\hline & & glucose 6-phosphate & 0.74 & 0.0299 \\
\hline & & fructose-6-phosphate & 0.79 & 0.0338 \\
\hline \multirow{3}{*}{$\begin{array}{l}\text { Cofactors and } \\
\text { Vitamins }\end{array}$} & Riboflavin Metabolism & flavin mononucleotide (FMN) & 0.71 & 0.0101 \\
\hline & Pantothenate and CoA Metabolism & pantothenate & 0.87 & 0.0299 \\
\hline & Hemoglobin and Porphyrin Metabolism & heme & 0.62 & 0.0433 \\
\hline \multirow{4}{*}{ Lipid } & Sterol & campesterol & 0.85 & 0.0243 \\
\hline & Sphingolipid Metabolism & sphingomyelin (d18:1/21:0, d17:1/22:0, d16:1/23:0)* & 1.11 & 0.0272 \\
\hline & Phospholipid Metabolism & 1,2-distearoyl-GPC (18:0/18:0) & 1.07 & 0.0276 \\
\hline & Lysolipid & 2-stearoyl-GPE $(18: 0)^{*}$ & 1.41 & 0.0315 \\
\hline Nucleotide & Purine and Pyrimidine Metabolism & methylphosphate & 0.86 & 0.0261 \\
\hline Peptide & Dipeptide & glycylvaline & 1.31 & 0.0055 \\
\hline \multirow[t]{2}{*}{ Xenobiotics } & \multirow[t]{2}{*}{ Food Component/Plant } & stachydrine & 0.69 & 0.0379 \\
\hline & & ergothioneine & 0.63 & 0.0407 \\
\hline
\end{tabular}

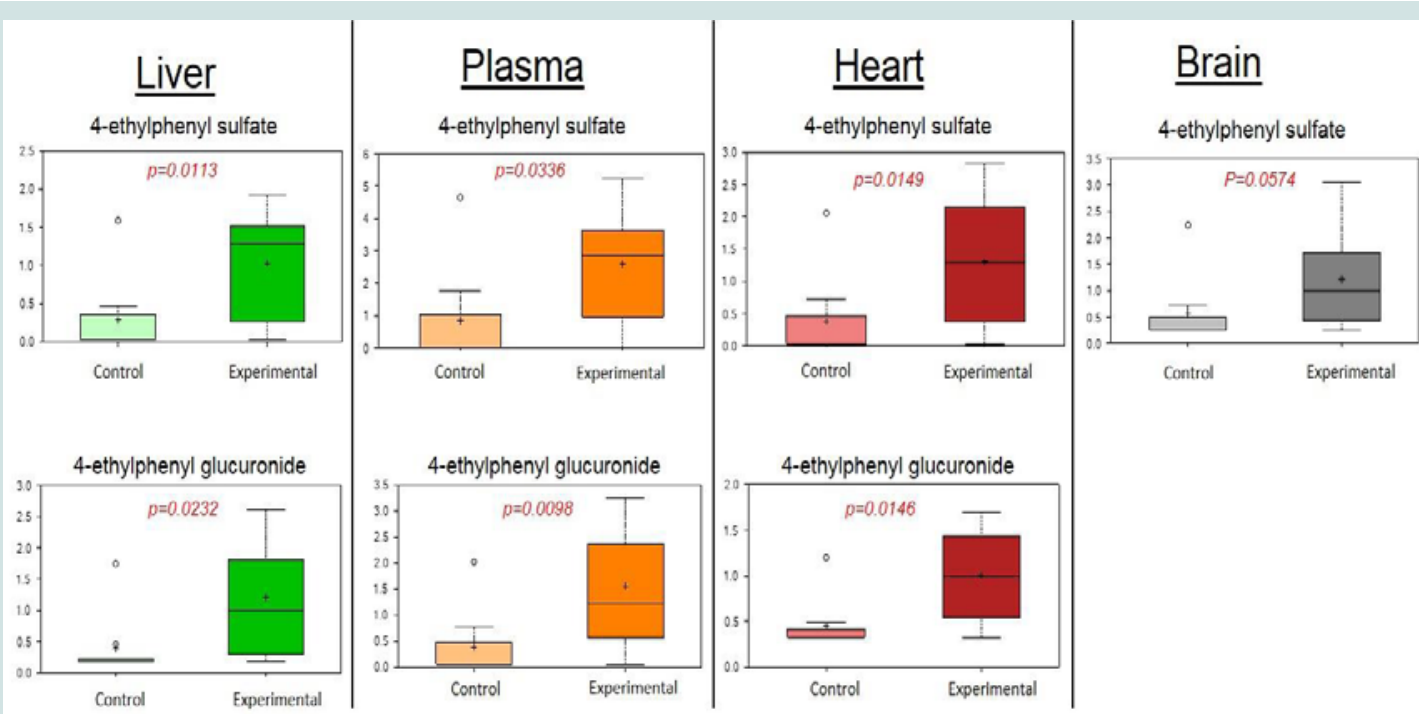

Figure 3: Levels of 4-EPS and 4-EPG in organs and plasma of experimental vs. control animals.

Data presented are mean+S.D. Welch's two sample t-tests. $\mathrm{N}=9$ animals per group. Experimental: oral application of $\mathrm{Pg}$, control vehicle alone. 
Citation: Ilievski V, Kinchen JM, Prabhu R, Rim F, Leoni L, et al. Experimental Periodontitis Results in Prediabetes and Metabolic Alterations in Brain, Liver and Heart: Global Untargeted Metabolomic Analyses. J Oral Bio. 2016; 3(1): 12.

ISSN: $2377-987 X$

Table 5: Metabolic differences in liver of experimental vs control animals. Fold change is expressed as Experimental/Control. $\mathrm{N}=9$ animals per group.

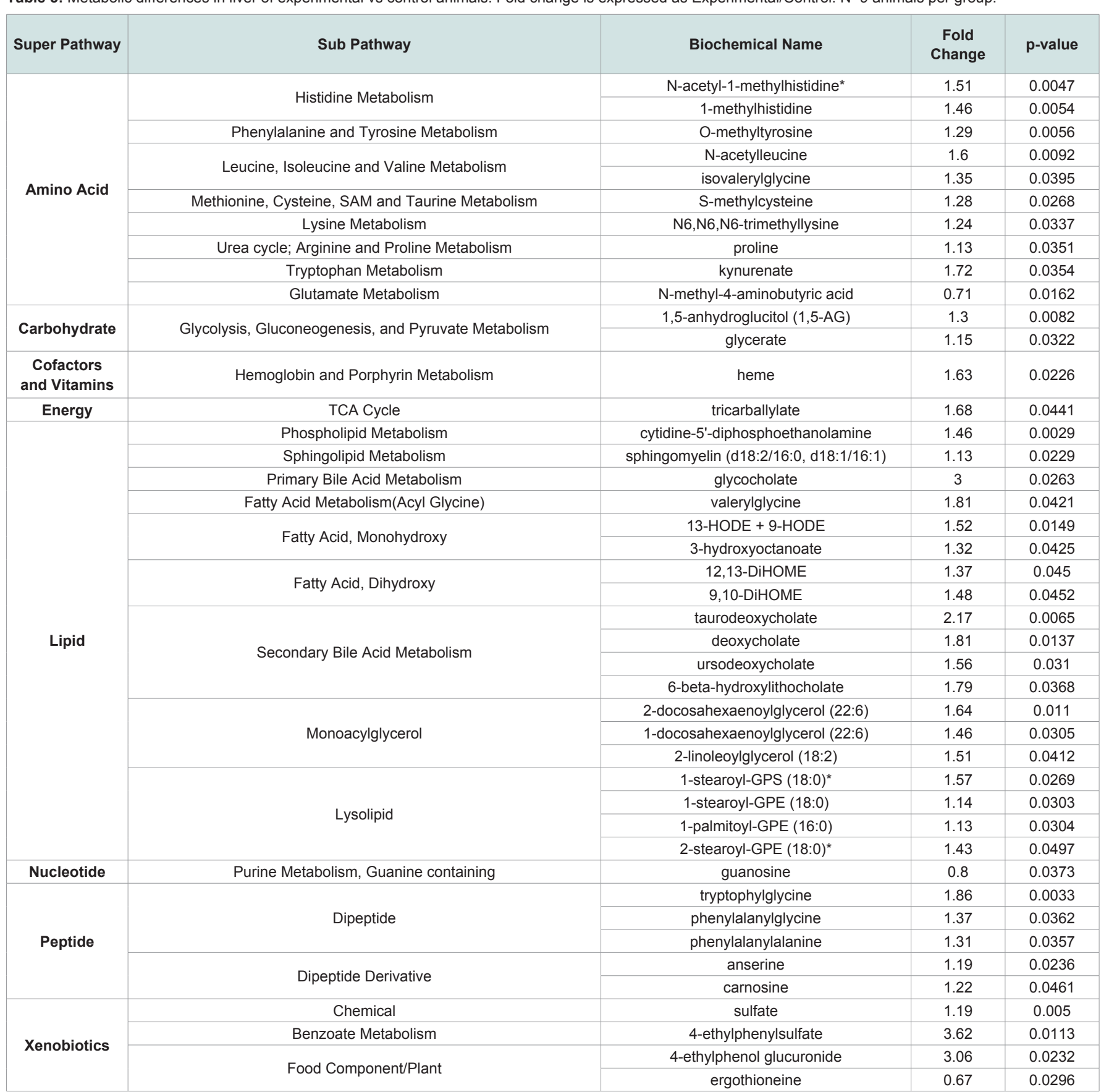

\section{Changes in biomarkers of oxidative stress in the liver were}

\section{evident in animals with periodontitis}

A number of changes in biomarkers of oxidative stress were observed in the liver of mice with periodontitis compared to controls (Table 5). Methionine sulfoxide and S-methylcysteine, products of methionine and cysteine oxidation respectively, were elevated in the liver of animals with periodontitis ( $\mathrm{p}=0.06$ and $\mathrm{p}=0.0268$ respectively), consistent with an increasingly oxidizing environment. Further, increases in the diols 9,10-DiHOME and 12,13-DiHOME ( $\mathrm{p}=0.0452$ and $\mathrm{p}=0.045$ respectively), derived from the opening of epoxides derived from linoleate, the diol 19,20-DiHDPA ( $\mathrm{p}=0.0821$ ), derived from the hydrolysis of the epoxide formed from docosahexaenoate or DHA, and 14/17-HDoHE ( $\mathrm{p}=0.2637$ ), an oxidation product of DHA were observed in the liver of animals with periodontitis. 9/13-HODE $(\mathrm{p}=0.0149)$, derived from the oxidation of the PUFA linoleate, was also elevated. 9/13-HODE is a biomarker of oxidative stress but also can be produced by lipoxygenases which produce proinflammatory eicosanoids. Glutathione levels, either reduced ( $\mathrm{GSH}, \mathrm{p}=0.6202$ ) or oxidized (GSSG, $\mathrm{p}=0.2061$ ) were not significantly changed, but anserine $(\mathrm{p}=0.0236)$ and carnosine $(\mathrm{p}=0.0461)$, both dipeptide metabolites of histidine with antioxidant function, were elevated 
Citation: Ilievski V, Kinchen JM, Prabhu R, Rim F, Leoni L, et al. Experimental Periodontitis Results in Prediabetes and Metabolic Alterations in Brain, Liver and Heart: Global Untargeted Metabolomic Analyses. J Oral Bio. 2016; 3(1): 12.

ISSN: $2377-987 X$

Table 6: Metabolic changes in the heart of experimental vs control animals. Fold change is expressed as Experimental/Control. $\mathrm{N}=9$ animals/group.

\begin{tabular}{|c|c|c|c|c|}
\hline Super Pathway & Sub Pathway & Biochemical Name & Fold changes & p-value \\
\hline \multirow{4}{*}{ Amino Acid } & Methionine, Cysteine, SAM and Taurine Metabolism & cystine & 1.39 & 0.0423 \\
\hline & Leucine, Isoleucine and Valine Metabolism & leucine & 1.13 & 0.0342 \\
\hline & Urea cycle; Arginine and Proline Metabolism & N-delta-acetylornithine & 0.87 & 0.0373 \\
\hline & Alanine and Aspartate Metabolism & $\mathrm{N}$-acetylalanine & 1.16 & 0.0235 \\
\hline \multirow{3}{*}{ Carbohydrate } & \multirow{2}{*}{ Glycolysis, Gluconeogenesis, and Pyruvate Metabolism } & 1,5-anhydroglucitol (1,5-AG) & 1.26 & 0.0089 \\
\hline & & pyruvate & 1.38 & 0.0496 \\
\hline & Advanced Glycation End-product & N6-carboxymethyllysine & 1.16 & 0.0292 \\
\hline $\begin{array}{l}\text { Cofactors and } \\
\text { Vitamins }\end{array}$ & Nicotinate and Nicotinamide Metabolism & $\begin{array}{l}\text { nicotinamide adenine dinucleotide reduced } \\
\qquad(\mathrm{NADH})\end{array}$ & 2.03 & 0.0228 \\
\hline \multirow{10}{*}{ Lipid } & \multirow{3}{*}{ Monoacylglycerol } & 2-oleoylglycerol (18:1) & 1.67 & 0.0132 \\
\hline & & 2-palmitoleoylglycerol (16:1) & 2.35 & 0.0392 \\
\hline & & 1-oleoylglycerol (18:1) & 1.98 & 0.0405 \\
\hline & \multirow{2}{*}{ Phospholipid Metabolism } & 1-palmitoyl-2-linoleoyl-GPC (16:0/18:2) & 0.84 & 0.0303 \\
\hline & & 1-palmitoyl-2-linoleoyl-GPE (16:0/18:2) & 0.85 & 0.0431 \\
\hline & Fatty Acid, Keto & 1-dihomo-linoleoylglycerol (20:2) & 2.13 & 0.0414 \\
\hline & Long Chain Fatty Acid & 10-nonadecenoate (19:1n9) & 1.45 & 0.0355 \\
\hline & Sphingolipid Metabolism & sphingomyelin (d18:2/16:0, d18:1/16:1) & 1.16 & 0.0375 \\
\hline & Fatty Acid, Dicarboxylate & undecanedioate & 1.24 & 0.0448 \\
\hline & Diacylglycerol & 1-palmitoleoyl-3-oleoyl-glycerol (16:1/18:1) & 1.73 & 0.0465 \\
\hline \multirow{2}{*}{ Xenobiotics } & Food Component/Plant & 4-ethylphenol glucuronide & 2.22 & 0.0146 \\
\hline & Benzoate Metabolism & 4-ethylphenylsulfate & 3.43 & 0.0149 \\
\hline
\end{tabular}

in the liver of animals with periodontitis, which suggests increased synthesis to meet an anti-oxidant demand.

\section{Signs of increasing inflammation are observed in the liver}

Several markers are useful in detecting subtle changes in inflammation. For example, changes in tryptophan metabolites can reflect an inflammatory state. Proinflammatory cytokines such as TNF $\alpha$ and INFy can activate conversion of tryptophan to kynurenine [16-18] which is catalyzed by indole amine 2,3-dioxygenase (IDO). Kynurenate was significantly increased $(\mathrm{p}=0.0354)$ (Table 5) and tryptophan and anthranilate were marginally increased $(\mathrm{p}=0.0842$ and 0.067 respectively) in the liver of animals with periodontitis. This may reflect increasing tryptophan availability or may reflect changes in indole amine 2,3-IDO expression or activity which has been shown to be induced in pathological conditions such as altered function of $\mathrm{T}$-cells at the level of antigen processing/presentation and during the early stage of primary biliary cirrhosis $[19,20]$.

Levels of biochemicals related to bile acid metabolism were also altered in the liver of animals with periodontitis; although cholesterol was not significantly changed, increases in 7-hydroxycholesterol $(\mathrm{p}=0.0519)$ suggest increased bile acid synthesis. Consistently, primary (e.g. glycocholate, $\mathrm{p}=0.0263$ and taurochenodeoxycholate, $\mathrm{p}=0.0542$ ) and secondary (e.g. deoxycholate, $\mathrm{p}=0.0137$ and urosodeoxycholate, $\mathrm{p}=0.031$ ) bile acids were also increased in animals with periodontitis. Increased bile acid production has been associated with liver disease such as nonalcoholic steatohepatitis [21] and cholesterol gallstone formation [22].

\section{Alterations in redox homeostasis were observed in the heart.}

Signs of changes in redox homeostasis were observed in the heart. For example, a significant increase in cysteine (oxidized form of cysteine) $(\mathrm{p}=0.0423)$ (Table 6) and a marginally significant increase in cysteine-glutathione disulfide $(\mathrm{p}=0.0771)$ were observed. Because of the ability of thiols to undergo redox reactions, cysteine has antioxidant properties. Thus, increases in these biochemicals suggest, that the heart may be under oxidative stress in animals with periodontitis. Given the importance of oxidative metabolism to 
Citation: Ilievski V, Kinchen JM, Prabhu R, Rim F, Leoni L, et al. Experimental Periodontitis Results in Prediabetes and Metabolic Alterations in Brain, Liver and Heart: Global Untargeted Metabolomic Analyses. J Oral Bio. 2016; 3(1): 12.

\section{ISSN: 2377-987X}

Table 7: Metabolic differences in plasma of experimental vs control animals. Fold change is expressed as Experimental/Control. $\mathrm{N}=9$ animals per group

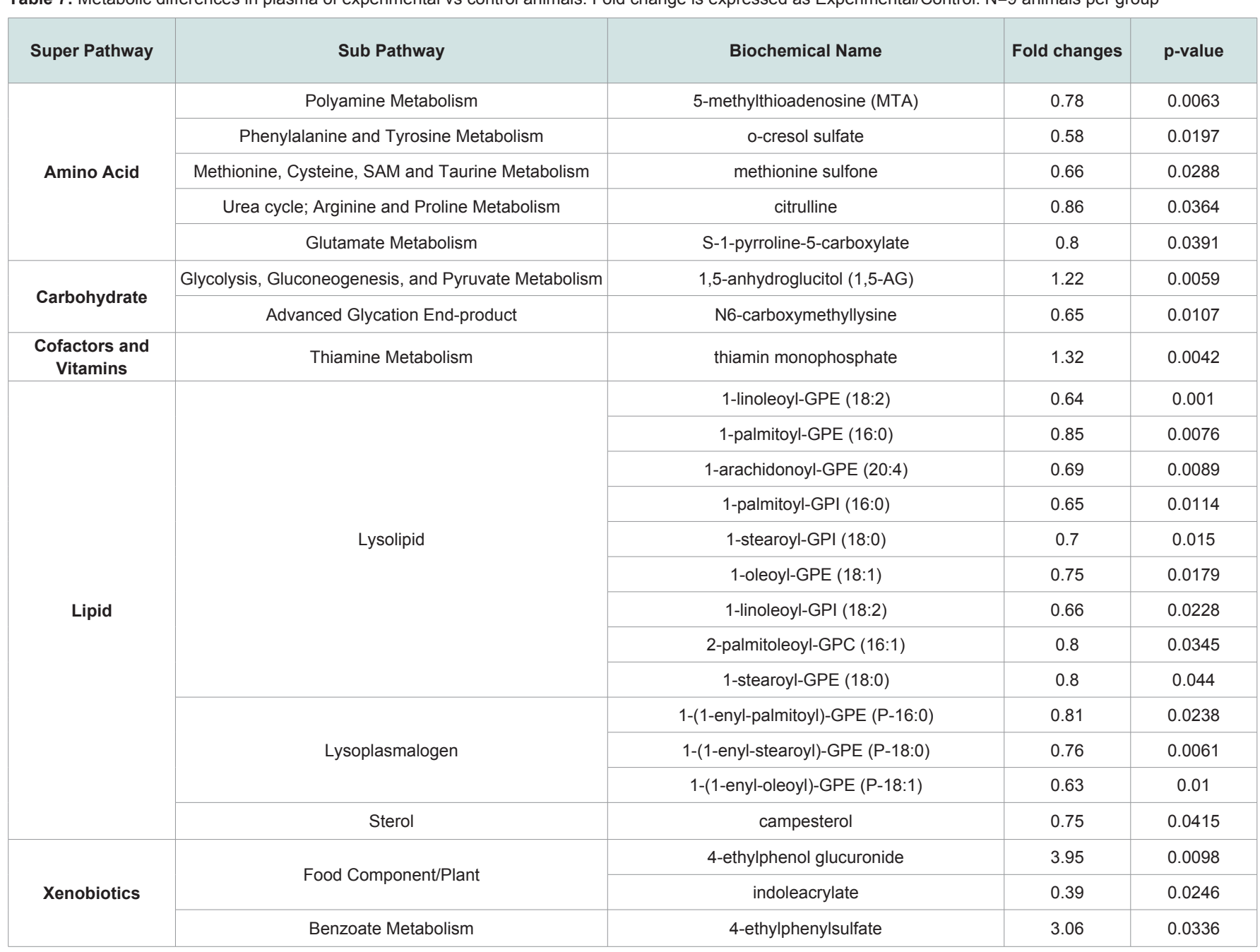

the heart, these changes may be early biomarkers of compromised cardiovascular function which is characteristic of diabetes.

Pyruvate levels in the heart were significantly elevated $(\mathrm{p}=0.0496)$. The heart typically relies on beta-oxidation for the majority of its energy, which shifts towards increased glycolytic metabolism seen during ischemic events $[23,24]$. Activated immune cells are highly glycolytic $[25,26]$ and infiltration of immune cells into the heart could contribute to changes in observed energy metabolism. The observed significant increase in reduced nicotinamide adenine dinucleotide, $\mathrm{NADH},(\mathrm{p}=0.0228)$ could reflect increased glycolytic metabolism. 1,5 -anhydroglucitol $(1,5-\mathrm{AG})$ levels are elevated $(\mathrm{p}=0.0089)$ in the heart also indicating an increased reliance on glycolysis, and an increase in the pentose metabolism product ribonate $(\mathrm{p}=0.0365)$, a ribose-derived product also is consistent with this concept.

In addition, the advanced glycation end-product (AGE) pathway product, N6-carboxymethyllysine was significantly increased $(\mathrm{p}=0.0292)$ in the heart.

Xanthurenate, a degradative product of kynurenine, is marginally increased in the heart of animals with periodontitis $(\mathrm{p}=0.052)$, which could also reflect a subtle change in inflammation.

Changes in the benzoate metabolite 4-ethylphenylsulfate (4EPS) in animals administered Pg may reflect changes in the

\section{microbiome.}

4-EPS and 4-ethylphenol glucuronide, derived from liver modifications of a microbiome-derived tyrosine metabolite 4-ethylphenol, were increased in all matrices in animals with periodontitis compared to control animals (Figure 3). 4-EPS is a bioactive metabolite linked to autism-like behavioral changes in mouse models [27], but little is known regarding the biological functions of this metabolite. Further studies to determine if this metabolite is derived from changes in the oral, intestinal, or both microbiomes would be of value.

\section{Plasma}

Decreased levels of the polyamine-related metabolites 5-methylthioadenosine (MTA) $(\mathrm{p}=0.0063)$, and citrulline $(\mathrm{p}=0.036)$, a urea cycle/arginine metabolite, were observed in animals with periodontitis compared with controls. In addition, multiple lysolipid 
Citation: Ilievski V, Kinchen JM, Prabhu R, Rim F, Leoni L, et al. Experimental Periodontitis Results in Prediabetes and Metabolic Alterations in Brain, Liver and Heart: Global Untargeted Metabolomic Analyses. J Oral Bio. 2016; 3(1): 12.

metabolites were significantly reduced in animals with periodontitis compared with controls (Table 7). Lysolipids are soluble in lipid bilayers and are capable of altering particular membrane functions [28]. For example, it has been shown that serum lysolipids can be readily incorporated into erythrocyte cell membranes and alter glucose transport [28].

In addition, a significant reduction in lysoplasmalogen occurred in animals with periodontitis (Table 7). Plasmalogens act as antioxidants to protect cells against reactive oxygen species produced during oxidative stress [29]. The decrease in plasmalogen content may reflect its function as a scavenger [30].

\section{Discussion}

Although the results from epidemiological and clinical studies demonstrate a close association between periodontitis and metabolic disorders such as metabolic syndrome and T2DM [3], the underlying metabolic alterations induced by periodontitis/ periodontal pathogens have not been elucidated. In the current study, we used the oral application of a periodontal pathogen, Pg, to induce chronic periodontitis (alveolar bone loss) and investigated changes in metabolism in critical organs distant from the oral cavity. All matrices (brain, liver, heart, plasma) that we examined exhibited altered biochemicals in response to oral application of Pg compared with control. This strongly suggests that repeated oral application of Pg alters metabolism in distinct patterns in organs distant from the oral cavity. This is the first time that such global change in vital organs has been reported. The results have important implications for the documented association between periodontal pathogen/bacterial products and a number of systemic diseases and conditions including the development of periodontitis and prediabetes.

Of particular interest were the changes in the metabolic profile in the brain in response to oral application of Pg. Major changes include reduced glucose utilization which is indicated by decreased glucose6-phosphate, fructose-6-phosphate and pyruvate levels. Decreased levels of 6-phosphogluconate suggest decreased pentose phosphate shunt activity which in turn leads to reduced NADPH which plays a role in preventing oxidative stress. Thus, when Pg is orally applied, host defense against oxidative stress may be compromised in the brain. Interestingly, decreased glucose availability was observed in the brains but not in plasma, heart or liver.

Polyamine metabolism was also significantly altered in the brain. Polyamines are ubiquitous molecules, highly regulated molecules that play critical roles in mammalian physiology including cell growth [31], cell division, differentiation, regulation of cell permeability and stability of cell membrane [32]. In addition, polyamines inhibit lipid peroxidation and prevent apoptosis [33]. Interestingly, they also modulate synaptic transmission in the central nervous system [34] and brain cells are extremely sensitive to reductions in polyamine levels [35]. Recently, polyamines were shown to protect memory impairment in an autophagy-dependent manner [36]. Thus, reduced polyamine levels in the brain may contribute to subtle changes in memory or cognition in animals with oral application of Pg in association with prediabetes.

The main energy source in the brain is glucose. However, during hypoglycemia, lactate from glycogen metabolism is used as a backup to ensure neuronal function [37]. We observed no significant difference in fasting glucose levels in plasma between control and periodontitis groups. However, biochemicals involved in the glycolysis pathway and amino (hexose) sugar metabolisms were significantly decreased, suggesting decreased glucose availability in the brain in animals with oral application of Pg.

Creatine, synthesized in the liver and kidney, is transported through the blood and taken up by tissues with high energy demands, such as the brain and skeletal muscle. In these tissues, they are converted to the high energy compound phosphocreatine and can be converted to creatinine [38]. Creatinine is degraded at a constant rate in the brain and secreted into the circulation [39]. We observed that creatinine was significantly reduced in the brain of animals with oral application of Pg but the implication of this finding is not known.

It is known that the plasma level of $1,5-\mathrm{AG}$ decreases during hyperglycemia and therefore can be used to determine glycemic control [40]. The level of 1,5-AG in blood is inversely related to the concentration of glucose and glycosylated hemoglobin levels in humans [41]. In the brain, 1,5-AG levels are elevated significantly compared with control animals. This may reflect low availability of glucose in the brain which is consistent with the observed decreased in glycolysis and amino (hexose) sugar metabolism in animals with oral application of Pg.

Sphingolipid metabolism increased in all matrices except plasma. Sphingolipids are essential membrane lipids that are enriched in lipid rafts and play an essential role in cell signaling [42] and also serve a structural role in myelinated neurons. Changes in sphingolipids can be indicative of alterations in these two processes and/or may also reflect changes in fatty acid biosynthesis or oxidation. It has been shown that animals injected with LPS exhibited increased levels of sphingomyelin and ceramide synthesis in the liver [43]. Thus increased levels of sphingomyelin may be due to Pg LPS. Increased sphingomyelin accumulation in the hippocampus has been reported in rats fed high-fat high-sugar chow with water with high-fructose content for 3 months [44]. Interestingly, our animals were fed a regular chow and yet exhibited increased sphingomyelin levels compared with controls.

In addition to the brain, significant alterations in response to oral application of Pg were noted in other organs. For example, increases in glycocholate, deoxycholate and ursodeoxycholate in the liver of animals with periodontitis suggest increased bile acid production. It has been reported that increased bile acid production is associated with liver disease such as nonalcoholic steatohepatitis [21] and cholesterol gallstone formation [22].

In the liver, increased biomarkers of oxidative stress such as methionine sulfoxide and S-methylcysteine, are increased in animals with oral application of Pg. This suggests that Pg or its products may disturb the physiological redox state resulting in production of peroxides and free radicals that damage cell components such as proteins, lipids, and DNA.

In contrast to the brain, the primary energy source for the heart is lipid oxidation (beta-oxidation). During ischemic damage or hypertrophy, however, energy utilization shifts towards glycolysis. Thus, elevated pyruvate levels may signify subtle metabolic changes 
Citation: Ilievski V, Kinchen JM, Prabhu R, Rim F, Leoni L, et al. Experimental Periodontitis Results in Prediabetes and Metabolic Alterations in Brain, Liver and Heart: Global Untargeted Metabolomic Analyses. J Oral Bio. 2016; 3(1): 12.

\section{ISSN: 2377-987X}

associated with cardiac muscle damage $[45,46]$.

Among the biochemicals which are significantly altered in animals with oral application of Pg compared with controls, 4-EPS and 1,5AG increased in all matrices. Increased levels of 4-EPS in animals reflect changes in liver-modified microbiome-derived products [27]. Whether 4-EPS is a derivative of Pg or intestinal bacteria due to a systemic disturbance resulting from oral application of Pg is not clear. The function of the bioactive metabolite 4-EPS is not known but has been linked to behavioral changes in mouse models [27] and thus is of interest in regards to cognition. 4-EPS is also found to be associated with percent lean mass in humans but periodontal status of these subjects were unknown [47].

Recently, the effect of oral administration of Pg on the gut microbiome has been reported $[48,49]$. Interestingly, repeated oral application of Pg resulted in changes in the composition of gut microbiota, but minimal amount of alveolar bone loss following 5 weeks of application [49]. A single application of Pg altered gut microbiota, increased serum endotoxin levels, and changed expression levels of proteins involved in intestinal permeability [48]. These results suggest that changes in the microbiota and/or intestinal permeability in response to periodontopathic bacteria may have metabolic consequences. Using a similar animal model and a similar time course as used in our current study, we previously noted no significant increase in plasma cytokine levels in animals that were administered LPS in the gingival sulci for 18 weeks, but there were significant differences in serum LPS levels [5]. In addition, these animals developed significant glucose intolerance and alveolar bone loss. Thus, it is still not clear if oral application of Pg influenced metabolic profiles via an alteration in gut microbiota or via the periodontal pocket to the systemic circulation.

There are an ample number of studies using experimental periodontitis animal models in which repeated oral application of Pg has been used to determine the effects of a periodontal pathogen in inducing periodontitis and/or direct effects on distant organs. However, methodologies have differed widely in terms of the duration of Pg application [49-56]. In most of these studies animals did not develop significant alveolar bone loss. In addition, the number of Pg present in the gingival sulci/periodontal pocket was unknown. As Pg may be present at low levels in healthy subgingival plaque/ biofilms, applying Pg to the oral cavity for short durations may not results in chronic periodontitis. Results from our previous studies indicate that application of LPS into the gingival sulci/periodontal pockets (without spilling out from the pockets) results in glucose intolerance and significant amount of alveolar bone loss [5]. Thus, monitoring glucose intolerance is one way to determine underlying systemic effects that relate to the development of periodontitis. In our current study, we applied Pg 3 times per week for 22 weeks based on the significant amount of alveolar bone loss (Figure 1) and the development of glucose intolerance (Table 1). In addition, all animals gained weight and consumed a normal amount of food (supplementary information) suggesting that they are apparently healthy.

In plasma, a decrease in 5-MTA in $(\mathrm{p}=0.0063)$ in animals with periodontitis compared with controls suggests declining polyamine production. This could relate to decreasing arginine availability. 1,5AG ( $p=0.0059)$ is inversely-associated with glucose levels in biological fluids such as saliva and serum $[41,57]$, thus, an increase in 1,5-AG levels could suggest subtle decreases in glucose availability. Trends toward decreases in lysolipids could reflect changes in signaling or demand for fatty acids to support beta-oxidation.

The purpose of the current study was to determine the effect of oral application of Pg on the metabolism in organs distant from the oral cavity using a global untargeted metabolic profiling in an experimental periodontitis model. The results provide a valuable resource of candidate metabolites and metabolic pathways to the scientific community to undertake further studies to unravel the consequences of oral application of periodontal pathogen(s) to a variety of systemic diseases and conditions, including prediabetes. Due to the large number of biochemicals that are altered upon oral application of $\mathrm{Pg}$, it is beyond the scope of the current study to describe the possible involvement and effects of all the changes that occurred. However, certain pathways are clearly evident. These include glycolysis and pentose phosphate pathways, amino (hexose) sugar and polyamine metabolism in the brain and in the liver, primary and secondary bile acid metabolism, fatty acid, tryptophan, and methionine metabolism. Cysteine metabolism and glycolysis pathways appear to be altered in the heart. In addition, alterations in polyamine, arginine, lysolipid and lysoplasmalogen pathways are evident in the plasma of animals with oral application of Pg compared with controls.

Periodontal disease is associated with a complex subgingival biofilm including Pg and other bacteria. The implications of bacterial interactions in the etiology of periodontitis were described [58]. The current study is limited by the use of Pg alone rather than a combination of several periodontal pathogens such as T. denticola, T. forsythia, F. nucleatum, and P. Intermedia to mimic a polymicrobial infection [59]. Studies using polymicrobial infections mimicking the complexity of the biofilm may shed light further into the influence of periodontal disease on systemic conditions.

In summary, oral application of Pg induces periodontitis, prediabetes, and alterations in various metabolic pathways in organs distant from the oral cavity. Further studies are needed to validate and expand on our findings and explore the relationship of periodontal pathogens to the large number of systemic diseases and conditions, such as dementia, atherosclerosis, and non-alcoholic fatty liver disease with which periodontitis has been shown to be associated in humans [60-62].

As a path forward, we hope scientists will investigate various metabolites and metabolic pathways which are altered by periodontal pathogens/bacterial products by examining gene expression as well as post-transcriptional modifications of enzymes, and other proteins involved in these pathways. In addition, more quantitative analysis of biochemicals involved in these metabolic pathways and off shoot pathways using targeted metabolic analyses may greatly help understand the mechanisms that are involved in altered metabolism. Identification of bacterial composition and bacterial products in 
Citation: Ilievski V, Kinchen JM, Prabhu R, Rim F, Leoni L, et al. Experimental Periodontitis Results in Prediabetes and Metabolic Alterations in Brain, Liver and Heart: Global Untargeted Metabolomic Analyses. J Oral Bio. 2016; 3(1): 12.

the gut, periodontal sites, and in different organs may be helpful in understanding the full systemic impact of periodontal pathogens/ products. Lastly, identification of mechanisms by which periodontal pathogens/products induce changes in metabolism and how these changes affect the development of prediabetes/T2DM may contribute to the prevention of the global diabetes epidemic and its severe complications.

\section{References}

1. Genco RJ, Grossi SG, Ho A, Nishimura F, Murayama Y (2005) A proposed model linking inflammation to obesity, diabetes, and periodontal infections. J Periodontol 76(11 Suppl): 2075-2084.

2. Saito T, Shimazaki $Y$ (2007) Metabolic disorders related to obesity and periodontal disease. Periodontol 2000 43: 254-266.

3. Watanabe K, Cho YD (2014) Periodontal disease and metabolic syndrome: a qualitative critical review of their association. Arch Oral Biol 59: 855-870.

4. Watanabe K, Petro BJ, Shlimon AE, Unterman TG (2008) Effect of periodontitis on insulin resistance and the onset of type 2 diabetes mellitus in Zucker diabetic fatty rats. J Periodontol 76: 1208-1216.

5. Ilievski V, Cho Y, Katwala P, Rodriguez H, Tulowiecka M, et al. (2015) TLR4 expression by liver resident cells mediates the development of glucose intolerance and insulin resistance in experimental periodontitis. PLoS One 10: e0136502.

6. Daviss B (2005) Growing pains for metabolomics. The Scientist 19: 25-28.

7. Dunn WB, Broadhurst D, Begley P, Zelena E, Francis-Mclntyre S, et al (2011) Procedures for large-scale metabolic profiling of serum and plasma using gas chromatography and liquid chromatography coupled to mass spectrometry. Nat Protoc 6: 1060-1083.

8. Jordan KW, Nordenstam J, Lauwers GY, Rothenberger DA, Alavi K, et al (2009) Metabolomic characterization of human rectal adenocarcinoma with intact tissue magnetic resonance spectroscopy. Dis Colon Rectum 52: 520 525.

9. Barnes VM, Kennedy AD, Panagakos F, Devizio W, Trivedi HM, et al. (2014) Global metabolomic analysis of human saliva and plasma from healthy and diabetic subjects, with and without periodontal disease. PLoS One 9 : e105181.

10. Marney LC, Kolwicz SC Jr, Tian R, Synovec RE (2013) Sample preparation methodology for mouse heart metabolomics using comprehensive two-dimensional gas chromatography coupled with time-of-flight mass spectrometry. Talanta 108: 123-130

11. Cheema AK, Pathak R, Zandkarimi F, Kaur P, Alkhalil L, et al. (2014) Liver metabolomics reveals increased oxidative stress and fibrogenic potential in gfrp transgenic mice in response to ionizing radiation. J Proteome Res 13 3065-3074.

12. Mamas M, Dunn WB, Neyses L, Goodacre R (2011) The role of metabolites and metabolomics in clinically applicable biomarkers of disease. Arch Toxico 85: 5-17.

13. Barnes VM, Ciancio SG, Shibly O, Xu T, Devizio W, et al. (2011) Metabolomics reveals elevated macromolecular degradation in periodontal disease. J Dent Res 90: 1293-1297.

14. Aimetti M, Cacciatore S, Graziano A, Tenori L (2012) Metabonomic analysis of saliva reveals generalized chronic periodontitis signature. Metabolomics 8: $465-474$.

15. Shin SY, Fauman EB, Petersen AK, Krumsiek J, Santos R, et al. (2014) An atlas of genetic influences on human blood metabolites. Nat Genet 46: 543 550 .

16. Ito H, Hoshi M, Ohtaki H, Taguchi A, Ando K, et al. (2010) Ability of IDO to attenuate liver injury in alpha-galactosylceramide-induced hepatitis model. $J$ Immunol 185: 4554-4560
17. Ohtaki $\mathrm{H}$, Ito $\mathrm{H}$, Ando $\mathrm{K}$, Ishikawa $\mathrm{T}$, Hoshi M, et al. (2014) Kynurenine production mediated by indoleamine 2,3-dioxygenase aggravates liver injury in HBV-specific CTL-induced fulminant hepatitis. Biochim Biophys Acta 1842: 1464-1471.

18. Cesario A, Rocca B, Rutella S (2011) The interplay between indoleamine 2,3-dioxygenase 1 (IDO1) and cyclooxygenase (COX)-2 in chronic inflammation and cancer. Curr Med Chem 18: 2263-2271.

19. Larrea E, Riezu-Boj Jl, Gil-Guerrero L, Casares N, Aldabe R, et al. (2007) Upregulation of indoleamine 2,3-dioxygenase in hepatitis $C$ virus infection. $J$ Virol 81: 3662-3666.

20. Oertelt-Prigione S, Mao TK, Selmi C, Tsuneyama K, Ansari AA, et al. (2008) Impaired indoleamine 2,3-dioxygenase production contributes to the development of autoimmunity in primary biliary cirrhosis. Autoimmunity 41 : 92-99.

21. Tanaka N, Matsubara T, Krausz KW, Patterson AD, Gonzalez FJ (2012) Disruption of phospholipid and bile acid homeostasis in mice with nonalcoholic steatohepatitis. Hepatology 56: 118-129.

22. Honda A, Yoshida T, Tanaka N, Matsuzaki Y, He B, et al. (1995) Increased bile acid concentration in liver tissue with cholesterol gallstone disease. J Gastroenterol 30: 61-66.

23. Lopaschuk GD (1997) Alterations in fatty acid oxidation during reperfusion of the heart after myocardial ischemia. Am J Cardiol 80: 11A-16A.

24. Jaswal JS, Keung W, Wang W, Ussher JR, Lopaschuk GD (2011) Targeting fatty acid and carbohydrate oxidation - a novel therapeutic intervention in the ischemic and failing heart. Biochim Biophys Acta 1813: 1333-1350.

25. Michalek RD, Rathmell JC (2011) The metabolic life and times of a T-cell. Immunol Rev 236: 190-202.

26. Macintyre AN, Rathmell JC (2013) Activated lymphocytes as a metabolic model for carcinogenesis. Cancer Metab 1: 5 .

27. Hsiao EY, McBride SW, Hsien S, Sharon G, Hyde ER, et al. (2013) Microbiota modulate behavioral and physiological abnormalities associated with neurodevelopmental disorders. Cell 155: 1451-1463.

28. Naderi S, Carruthers A, Melchior DL (1989) Modulation of red blood cell sugar transport by lyso-lipid. Biochim Biophys Acta 985: 173-183.

29. Reiss D, Beyer K, Engelmann B (1997) Delayed oxidative degradation of polyunsaturated diacyl phospholipids in the presence of plasmalogen phospholipids in vitro. Biochem J 323: 807-814

30. Dudda A, Spiteller G, Kobelt F (1996) Lipid oxidation products in ischemic porcine heart tissue. Chem Phys Lipids 82: 39-51.

31. Pegg AE (2009) Mammalian polyamine metabolism and function. IUBMB Life 61: $880-894$

32. Schuber F (1989) Influence of polyamines on membrane functions. Biochem J 260: 1-10.

33. Thomas T, Thomas TJ (2001) Polyamines in cell growth and cell death: molecular mechanisms and therapeutic applications. Cell Mol Life Sci 58: 244-258.

34. Halonen T, Sivenius J, Miettinen R, Halmekytö M, Kauppinen R, et al. (1993) Elevated seizure threshold and impaired spatial learning in transgenic mice with putrescine overproduction in the brain. Eur J Neurosci 5: 1233-1239.

35. Slotkin TA, Bartolome J, Persons D, Whitmore WL (1984) Polyamines in brain and heart of the neonatal rat: effects of inhibitors of ornithine decarboxylase and spermidine synthase. Life Sci 35: 1125-1131.

36. Gupta VK, Scheunemann L, Eisenberg T, Mertel S, Bhukel A, et al. (2013) Restoring polyamines protects from age - induced memory impairment in an autophagy-dependent manner. Nat Neurosci 16: 1453-1460.

37. Falkowska A, Gutowska I, Goschorska M, Nowacki P, Chlubek D, et al (2015) Energy metabolism of the brain, including the cooperation between astrocytes and neurons, especially in the context of glycogen metabolism. Int J Mol Sci 16: 25959-25981. 
Citation: Ilievski V, Kinchen JM, Prabhu R, Rim F, Leoni L, et al. Experimental Periodontitis Results in Prediabetes and Metabolic Alterations in Brain, Liver and Heart: Global Untargeted Metabolomic Analyses. J Oral Bio. 2016; 3(1): 12.

38. Howard AD, Moore J Jr, Welch PG, Gouge SF (1989) Analysis of the quantitative relationship between anemia and chronic renal failure. Am J Med Sci 297: 309-313.

39. Stöckler S, Holzbach U, Hanefeld F, Marquardt I, Helms G, et al. (1994) Creatine deficiency in the brain: a new, treatable inborn error of metabolism. Pediatr Res 36: 409-413.

40. Dungan KM (2008) 1,5-anhydroglucitol (GlycoMark) as a marker of short-term glycemic control and glycemic excursions. Expert Rev Mol Diagn 8: 9-19.

41. Mook-Kanamori DO, Selim MM, Takiddin AH, Al-Homsi H, Al-Mahmoud KA et al. (2014) 1,5-anhydroglucitol in saliva is a noninvasive marker of shortterm glycemic control. J Clin Endocrinol Metab 99: E479-E483.

42. Simons K, Toomre D (2000) Lipid rafts and signal transduction. Nat Rev Mol Cell Biol 1: 31-39.

43. Memon RA, Holleran WM, Moser AH, Seki T, Uchida Y, et al. (1998) Endotoxin and cytokines increase hepatic sphingolipid biosynthesis and produce lipoproteins enriched in ceramides and sphingomyelin. Arterioscler Thromb Vasc Biol 18: 1257-1265.

44. Stranahan AM, Cutler RG, Button C, Telljohann R, Mattson MP (2011) Dietinduced elevations in serum cholesterol are associated with alterations in hippocampal lipid metabolism and increased oxidative stress. J Neurochem 118: 611-615.

45. Das DK, Engelman RM, Rousou JA, Breyer RH (1987) Aerobic vs anaerobic metabolism during ischemia in heart muscle. Ann Chir Gynaecol 76: 68-76.

46. Kolwicz SC Jr, Tian R (2011) Glucose metabolism and cardiac hypertrophy. Cardiovasc Res 90: 194-201.

47. Lustgarten MS, Price LL, Phillips EM, Kirn D, Mills J, et al. (2013) Serum predictors of percent lean mass in young adults. J Strength Cond Res [Epub ahead of print]

48. Nakajima M, Arimatsu K, Kato T, Matsuda Y, Minagawa T, et al. (2015) Oral administration of $P$. gingivalis induces dysbiosis of gut microbiota and impaired barrier function leading to dissemination of enterobacteria to the liver. PLoS One 10: e0134234.

49. Arimatsu K, Yamada H, Miyazawa H, Minagawa T, Nakajima M, et al. (2014) Oral pathobiont induces systemic inflammation and metabolic changes associated with alteration of gut microbiota. Sci Rep 4: 4828.

50. Lalla E, Lamster IB, Hoffman MA, Bucciarelli L, Jerud AP, et al. (2003) Oral infection with a periodontal pathogen accelerates early atherosclerosis in apolipoprotein E-null mice. Arterioscler Thromb Vasc Biol 23: 1405-1411.
51. Baker PJ, DuFour L, Dixon M, Roopenian DC (2000) Adhesion molecule deficiencies increase Porphyromonas gingivalis-induced alveolar bone loss in mice. Infect Immun 68: 3103-3107.

52. Kuula H, Salo T, Pirilä E, Tuomainen AM, Jauhiainen M, et al. (2009) Loca and systemic responses in matrix metalloproteinase 8-deficient mice during Porphyromonas gingivalis-induced periodontitis. Infect Immun 77: 850-859.

53. Polak D, Wilensky A, Shapira L, Halabi A, Goldstein D, et al. (2009) Mouse model of experimental periodontitis induced by Porphyromonas gingivalis/ Fusobacterium nucleatum infection: bone loss and host response. J Clin Periodontol 36: 406-410.

54. Sasaki H, Okamatsu Y, Kawai T, Kent R, Taubman M, et al. (2004) The interleukin-10 knockout mouse is highly susceptible to Porphyromonas gingivalis-induced alveolar bone loss. J Periodontal Res 39: 432-441.

55. Burns E, Bachrach G, Shapira L, Nussbaum G (2006) Cutting edge: TLR2 is required for the innate response to Porphyromonas gingivalis: activation leads to bacterial persistence and TLR2 deficiency attenuates induced alveolar bone resorption. J Immunol 177: 8296-8300.

56. Wilensky A, Gabet Y, Yumoto H, Houri-Haddad Y, Shapira L (2005) Threedimensional quantification of alveolar bone loss in Porphyromonas gingivalisinfected mice using micro-computed tomography. J Periodontol 76: 12821286.

57. Buse JB, Freeman JL, Edelman SV, Jovanovic L, McGill JB (2003) Serum 1,5-anhydroglucitol (GlycoMark): a short-term glycemic marker. Diabetes Technol Ther 5: 355-363.

58. Kuramitsu HK, He X, Lux R, Anderson MH, Shi W (2007) Interspecies interactions within oral microbial communities. Microbiol Mol Biol Rev 71: 653-670.

59. Rivera MF, Lee JY, Aneja M, Goswami V, Liu L, et al. (2013) Polymicrobial infection with major periodontal pathogens induced periodontal disease and aortic atherosclerosis in hyperlipidemicApoE(null) mice. PLoS One 8: e57178.

60. Noble JM, Borrell LN, Papapanou PN, Elkind MS, Scarmeas N, et al. (2009) Periodontitis is associated with cognitive impairment among older adults: analysis of NHANES-III. J Neurol Neurosurg Psychiatry 80: 1206-1211.

61. Söder PO, Söder B, Nowak J, Jogestrand T (2005) Early carotid atherosclerosis in subjects with periodontal diseases. Stroke 36: 1195-1200.

62. Yoneda M, Naka S, Nakano K, Wada K, Endo H, et al. (2012) Involvement of a periodontal pathogen, Porphyromonas gingivalis on the pathogenesis of non-alcoholic fatty liver disease. BMC Gastroenterol 12: 16.

\section{Acknowledgements}

This work was supported by NIH R01 DE021405 (KW). 